\title{
Los cariotipos de Cologania grandiflora y Erythrina americana (Leguminosae- Papilionoideae-Phaseoleae) de la Reserva Ecológica del Pedregal de San Ángel, México
}

\section{Karyotypes of Cologania grandiflora and Erythrina americana (Leguminosae-Papilionoideae- Phaseoleae) of Reserva Ecológica del Pedregal de San Ángel, Mexico}

\author{
Fernando Tapia-Pastrana* y Anahid Jiménez-Salazar \\ Laboratorio de Genecología, División de Estudios de Posgrado e Investigación, Facultad de Estudios Superiores Zaragoza, Universidad Nacional \\ Autónoma de México. Apartado postal 9-020, 15000 México, D.F., México. \\ *Correspondencia: pasfer@unam.mx
}

\begin{abstract}
Resumen. Se analizaron citogenéticamente células provenientes de meristemos radiculares de 2 leguminosas, Cologania grandiflora y Erythrina americana, que en la actualidad están incluidas en la flora de la Reserva Ecológica del Pedregal de San Ángel, México, D. F., mediante una técnica de extendido en superficie y secado al aire para determinar los números cromosómicos somáticos. Por vez primera se obtuvieron la morfología cromosómica y otras características cuantitativas de los cariotipos en C. grandiflora $(2 n=44=26 \mathrm{~m}+18 \mathrm{sm})$ y en E. americana $(2 n=42=36 \mathrm{~m}$ $\left.+4 \mathrm{sm}+2 \mathrm{st}^{\mathrm{sat}}\right)$, primeras también en ambos géneros. Cologania y Erythrina se reconocen como poliploides estabilizados y el hallazgo de un único par de cromosomas con satélites (dominancia nucleolar) en las especies estudiadas aquí, favorece la opinión de un origen alopoliploide para estos taxa.
\end{abstract}

Palabras clave: alopoliploide, cariotipo, Cologania, Erythrina, satélites, secado al aire.

\begin{abstract}
Meristematic root cells from Cologania grandiflora and Erythrina americana from Reserva Ecológica del Pedregal de San Ángel, Distrito Federal, Mexico, were analyzed cytogenenetically using a surface-spreading and airdrying method. The somatic chromosome numbers were determined. Chromosome morphology and others quantitative features of the karyotypes obtained for first time in C. grandiflora $(2 n=44=26 \mathrm{~m}+18 \mathrm{sm})$ and E. americana $(2 n=42=$ $\left.36 \mathrm{~m}+4 \mathrm{sm}+2 \mathrm{st}^{\mathrm{sat}}\right)$ and also the first in both genera. Cologania and Erythrina are recognized as stabilized polyploids and the finding of just one pair of chromosomes with satellites (nucleolar dominance) in the species analyzed here supports the view of allopolyploid origin of these taxa.
\end{abstract}

Key words: allopolyploid, karyotype, Cologania, Erythrina, satellites, surface-spreading and air-drying.

\section{Introducción}

La Reserva Ecológica del Pedregal de San Ángel (REPSA), localizada dentro de los terrenos del campo central de la Universidad Nacional Autónoma de México, en México, D. F., exhibe como tipo de vegetación dominante un matorral xerófilo de Senecio praecox (Senecionetum praecosis), y desde el siglo XIX el sitio se considera de alta riqueza florística (Rzedowski, 1954; Valiente-Banuet y Luna, 1990). Está ubicada en altitudes que oscilan entre 2200 y 2277 m snm y sobre un área de 237.3 ha; presenta una diversidad de hábitats que albergan cerca de 350 especies de plantas, muchas de ellas endémicas del Pedregal (Portal de la Reserva Ecológica Pedregal de San

Recibido: 17 agosto 2010; aceptado: 14 febrero 2011
Ángel. 2007. http://www.cic-ctic unam.mx:31101/reserva ecológica).

La flora de la REPSA ha experimentado cambios en su composición original, como se aprecia al comparar los listados florísticos realizados durante el siglo XX (Rzedowski, 1954; Valiente-Banuet y Luna, 1990). La introducción de especies ruderales (asociadas a perturbación) y de algunas ornamentales han contribuido con este fenómeno (Valiente-Banuet y Luna, 1990; Kelly y Delgado-Salinas, s/f, http://www.arboles.org/).

Diversas especies de la familia Leguminosae incluidas en 15 géneros (Valiente-Banuet y Luna, 1990) contribuyen en la actualidad a la riqueza florística de la REPSA; entre ellas, Cologania grandiflora Rose y Erythrina americana Miller.

Cologania Kunth (Papilionoideae, Phaseoleae, Glycininae) es un género americano que se extiende desde Estados Unidos, México, centro y sur América hasta 
Argentina central (Musicante y Galetto, 2008). El grupo incluye 10 especies, todas presentes en México, país que cuenta con 7 endemismos (Sousa y Delgado, 1998; Lee y Hymowitz, 2001). Cologania grandiflora es una especie subendémica del Valle de México que se encuentra en el listado de especies amenazadas elaborada por Rzedowski y Rzedowski (1993).

Recuentos citogenéticos previos en Cologania registran un número cromosómico haploide $n=22$ para $C$. ovalifolia (Hunziker et al., 1985) que confirma el $x=11$ hallado hasta ahora en 4 especies del género (Lackey, 1980; Goldblatt, 1981).

Erythrina L. (Papilionoideae, Phaseoleae, Erythrininae) comprende 112 especies distribuidas principalmente a través de las regiones tropicales y algunas templado cálidas (Krukoff y Barneby, 1974). Las especies son principalmente árboles o arbustos, pero 10 son hierbas perennes (Neill, 1988). En México se encuentran 28 especies de las cuales 17 son endémicas (Sousa y Delgado, 1998). Erythrina americana Miller (= E. coralloides A. DC.) Secc. Erythrina, Krukoff et Barneby, es un árbol pequeño que se distribuye desde Arizona hasta México, donde comúnmente se conoce como colorín y se le utiliza como árbol de ornato (Martínez, 1979; Kelly, L.M. y A. Delgado Salinas, s/f. http://www.arboles.org/).

Se conocen los números cromosómicos de alrededor del $75 \%$ de las especies de Erythrina. La mayoría tienen un número básico $x=21$ y diploide $2 n=2 x=42$, aunque existen registros de poliploidía $(2 n=4 x=84)$ o diferentes niveles de ploidía en 6\% de las especies (Lackey, 1980; Goldblatt, 1981; Neill, 1988). Con excepción del número cromosómico y del estudio sobre el comportamiento meiótico en algunas especies (Cruz et al., 1976; Jalil et al., 1982; Neill, 1988), no existe información disponible sobre cariotipos para las especies del género. En un intento para comparar citogenéticamente las especies de Erythrina se evaluó la frecuencia de quiasmas con resultados limitados, pues los índices de recombinación permitieron distinguir subgéneros, pero no facultó la separación en secciones (Forni-Martins y Cruz, 1996).

A pesar de que los números cromosómicos han sido de gran valor en la revisión y mejoramiento de la clasificación en la familia Leguminosae y en el entendimiento de su evolución (Goldblatt, 1981; Poggio et al., 2008), y de que tanto en Cologania como en Erythrina existen recuentos para una buena proporción de sus especies, es notoria la falta de estudios citogenéticos que detallen la posición del centrómero, número, tipo y posición de zonas organizadoras del nucléolo, tamaño de los cromosomas y longitud cromosómica total, entre otras características de los complementos cromosómicos, lo cual impide acceder a una de las fuentes de información más valiosa para el análisis de los mecanismos evolutivos implicados en la especiación y diversidad de las plantas.

El objetivo de este trabajo es analizar las características citogenéticas de C. grandiflora y E. americana, obtener sus cariotipos y contribuir al conocimiento de la biología básica de 2 de las leguminosas que conforman la flora de la Reserva Ecológica del Pedregal de San Ángel, México, D. F.

\section{Materiales y métodos}

Se colectaron frutos de C. grandiflora y E. americana en áreas de amortiguamiento de la Reserva Ecológica del Pedregal de San Ángel, en México, D. F., al sur de la cuenca del Valle de México (entre $19^{\circ} 20^{\prime} 2^{\prime \prime}$ y $19^{\circ} 13^{\text {' }}$ $45^{\prime \prime} \mathrm{N}$; entre $99^{\circ} 08^{\prime} 26^{\prime \prime}$ y $99^{\circ} 14^{\prime} 3$ " O) en el mes de septiembre de 2008: zona A5 (Paseo de las Esculturas), zona A8 (Biológicas) y zona A10 (Jardín Botánico). Semillas seleccionadas al azar, provenientes al menos de 3 individuos de las áreas de colecta fueron puestas a germinar a temperatura ambiente y en luz natural en cajas de Petri con algodón humedecido con agua destilada. Las raíces de 1-2 $\mathrm{cm}$ de largo fueron cortadas y pretratadas en 8-hidroxiquinoleína $0.002 \mathrm{M}$ durante 5 horas a temperatura ambiente. Posteriormente se fijaron en solución Farmer (etanol-ácido acético, 3:1). Para la obtención de los cromosomas en metafase se siguió el método de secado al aire descrito en Tapia-Pastrana y Mercado-Ruaro (2001). Los cromosomas se tiñieron con Giemsa al 10\% y las preparaciones se estabilizaron empleando Entellan Nuevo (Merck) como medio de montaje. Los mejores campos se fotografiaron en un microscopio óptico Zeiss Axioscop usando película Kodak Technical Pan.

Se utilizó un vernier digital (Mitutoyo Digimatic Caliber CD-G”BS) para establecer las tallas cromosómicas sobre 4 fotografías de placas en metafase con la misma magnificación. Para obtener la fórmula cariotípica se aplicó el sistema propuesto por Levan et al. (1964). La proporción de la suma total de longitudes de brazos cortos respecto a la suma total de longitudes cromosómicas como indicador de simetría o asimetría de un cariotipo (T. F.\%) se realizó según Sinha y Roy (1979).

\section{Resultados}

Cologania grandiflora. Se revisaron 142 células en metafase que mostraron un número cromosómico diploide $2 n=$ 44, siendo éste el primer registro para esta especie. Los cromosomas exhibieron una talla cromosómica promedio cercana a $2 \mu \mathrm{m}$ y en su cariotipo únicamente se observaron cromosomas metacéntricos y submetacéntricos. Un par de estos últimos muestra una constricción secundaria y 
notoria porción satélite sobre los brazos cortos (Figs. 1A y 2). A pesar de la ausencia de cromosomas subtelocéntricos el cariotipo es ligeramente asimétrico (T. F.\%= 39.53). Erythrina americana. Se revisaron 244 células en metafase y en todos ellos se registró un número cromosómico $2 n=$ 42, mismo que confirma el que Atchison (1947).obtuvo previamente. La talla cromosómica promedio, aunque pequeña (ca. $1.47 \mu \mathrm{m}$ ), no impidió el reconocimiento individual de los cromosomas. El cariotipo exhibe una predominancia de cromosomas metacéntricos y submetacéntricos y un solo par de cromosomas subtelocéntricos, los más pequeños del complemento, mismos que son portadores de una constricción secundaria y micro satélite en el brazo corto (Figs. 1B y 3). El cariotipo es ligeramente asimétrico (T. F.\%= 39.68).

Los datos cuantitativos sobre la morfología cromosómica de ambas especies se resumen en el Cuadro 1. Los cariotipos y complementos cromosómicos se muestran en las figuras 1-3.

\section{Discusión}

La tribu Phaseoleae ha sido observada como un agrupamiento citogenético bastante uniforme y con excepción de Erythrininae $(x=21)$, muestra un número cromosómico básico predominante $x=11(n=11 ; 2 n=22)$ (Goldblatt, 1981). Cologania y Erythrina representan algunos de los escasos grupos poliploides. En la tribu, la mayoría de los cromosomas son de talla pequeña (Lackey, 1980). Nuestros resultados confirman tales características.

Por otro lado, ya que Cologania y Erythrina, considerados poliploides estabilizados, poseen elevados números cromosómicos y tallas cromosómicas pequeñas, la investigación citogenética sólo ha considerado abordar el registro de números cromosómicos, lo que se traduce en la ausencia de cariotipos (Lackey, 1980). La presente investigación muestra que la obtención de abundantes células intactas con una distribución óptima de los cromosomas posibilita el análisis adecuado de los complementos cromosómicos y la elaboración de cariotipos en $C$. grandiflora y E. americana (Figs. 2 y 3 ).

Las formulas cromosómicas de C. grandiflora $(26 \mathrm{~m}+$ $18 \mathrm{sm})$ y E. americana $(36 \mathrm{~m}+4 \mathrm{sm}+2 \mathrm{st}$ sat $)$ junto con el índice de asimetría (Cuadro 1) señalan cariotipos ligeramente asimétricos y sólo E. americana presenta un par de cromosomas subtelocéntricos con microsatélites y cuya frecuente asociación con el nucleolo en células en prometafase permite suponer que son portadores de la región NOR -cromosomas nucleolares-(Fig. 4). En relación con la talla cromosómica, ambas especies poseen cromosomas de talla pequeña, donde los más grandes no superan los 3 $\mu \mathrm{m}$ y los pequeños se encuentran cercanos a $1 \mu \mathrm{m}$, siendo ligeramente mayores en $C$. grandiflora lo que aunado a su mayor numero cromosómico le confiere una mayor longitud cromosómica total haploide (Cuadro 1).

En estudios previos sobre el tamaño y morfología cromosómica de otras especies incluidas en Phaseoleae se registran rangos cromosómicos similares a los registrados aquí, con cariotipos donde predominan cromosomas metacéntricos y submetacéntricos con escasos o nulos subtelocéntricos (Souza y Benko-Iseppon, 2004; Sede et al., 2006). Llama particularmente la atención, la talla cromosómica registrada en géneros diploides como Camptosema Hook. et Arn., caracterizado por $2 n=22$, y Galactia P. Browne y Collaea DC., descritos con $2 n=20$ (Sede et al., 2006). En estos géneros las longitudes cromosómicas totales son similares a las obtenidas en esta investigación para $C$. grandiflora y E. americana, con la salvedad de que estas últimas son poliploides.

En Leguminosae comienza a vislumbrarse la correlación inversa entre el nivel de ploidía y la talla cromosómica total: las especies diploides muestran longitudes cromosómicas mayores que las tetraploides. Esto es evidente en géneros como Acacia (Sharma y Sen, 2002), Crotalaria (Atchison, 1950; Palomino y Vázquez, 1991; Cotias de Oliveira y Aguiar-Perecin, 1999; Almada et al., 2006), Lathyrus y Vicia (Sharma, 1970; Sharma y Sharma, 1984). Al respecto, la evaluación cariotípica de un mayor número de especies en los paleopoliploides Cologania y Erythrina será necesaria antes de llegar a conclusiones más certeras sobre este interesante fenómeno.

Finalmente, el inusual $x=21$ consistentemente hallado en Erythrina, ha sido interpretado como legado de un alopoliploide ancestral derivado de plantas con $n=11+n=$ 10 (Senn, 1938; Atchison, 1947; Lackey, 1980), aunque también se ha sugerido un origen hipotetraploide $n=(11 \mathrm{X}$

Cuadro 1. Características citogenéticas de las especies estudiadas

\begin{tabular}{lcccccc}
\hline \multicolumn{1}{c}{ Especie } & $2 n$ & $F C$ & LCT & TCP & Rango & T.F. \% \\
\hline Cologania grandiflora & 44 & $26 \mathrm{~m}+18 \mathrm{sm}$ & 41.84 & 1.90 & $2.45-1.44$ & 39.53 \\
Erythrina americana & 42 & $\begin{array}{c}36 \mathrm{~m}+4 \mathrm{sm}+ \\
2 \mathrm{st} \text { sat }\end{array}$ & 31.00 & 1.47 & $2.03-0.87$ & 39.68 \\
\hline
\end{tabular}

$2 n$, número cromosómico diploide; FC, fórmula cariotípica; LCT, longitud cromosómica total haploide en $\mu$ m; TCP, talla cromosómica promedio en $\mu \mathrm{m}$; Rango, rango de longitud cromosómica en $\mu \mathrm{m}$; T.F.\%, índice de asimetría. 


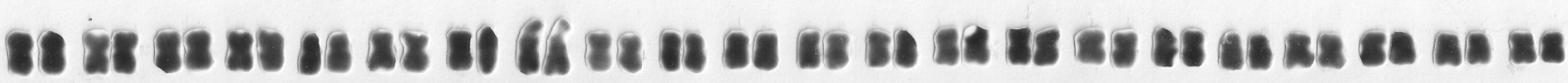 A

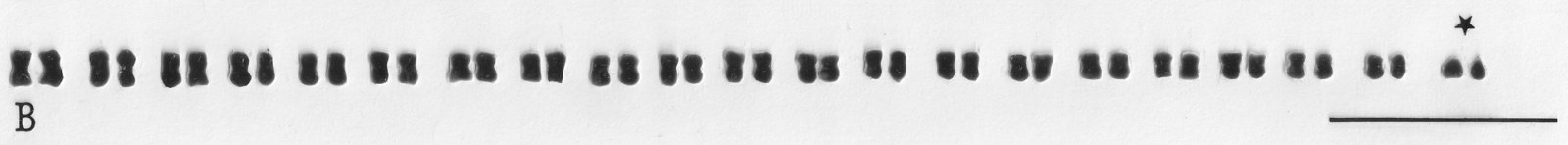

Figura 1. Cariotipos en metafase mitótica. A. Cologania grandiflora $(2 n=4 x=44)$; B. Erythrina americana, $2 n=4 x=42$. Los cromosomas están alineados por el centrómero y numerados en orden decreciente de tamaño. Con asterisco $(*)$, cromosomas con satélite; escala, $10 \mu \mathrm{m}$.

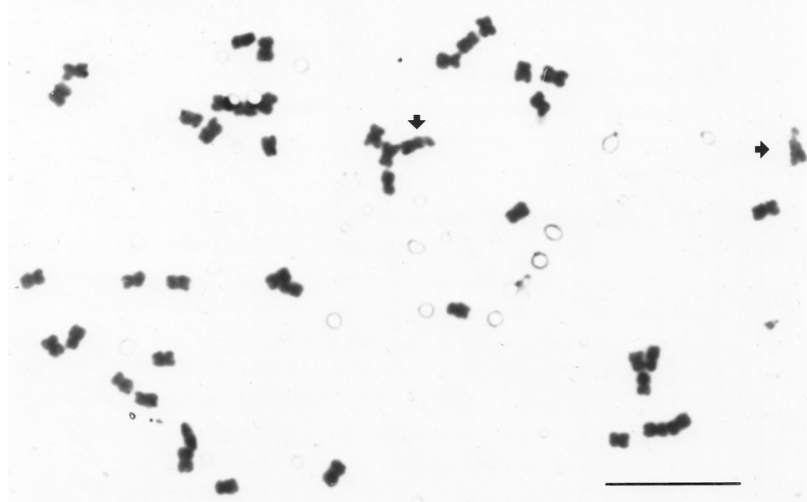

Figura 2. Complemento cromosómico en metafase mitótica de Cologania grandiflora. Las flechas señalan los cromosomas con satélites. Escala, $10 \mu \mathrm{m}$.

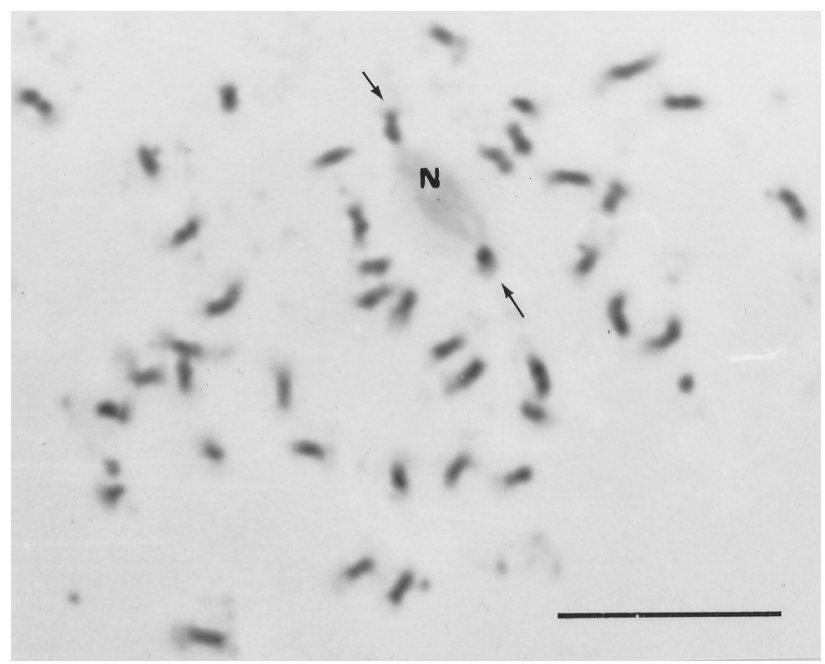

Figura 4. Prometafase de Erythrina americana. Las flechas señalan los cromosomas nucleolares adheridos al nucleolo (N), el cual se observa bajo fuerte tensión. Escala, $10 \mu \mathrm{m}$.

2) - 1 (Lewis, 1974; Goldblatt, 1981). La existencia en $E$. americana de un único par cromosómico subtelocéntrico que además es portador de satélites apoyaría la primera

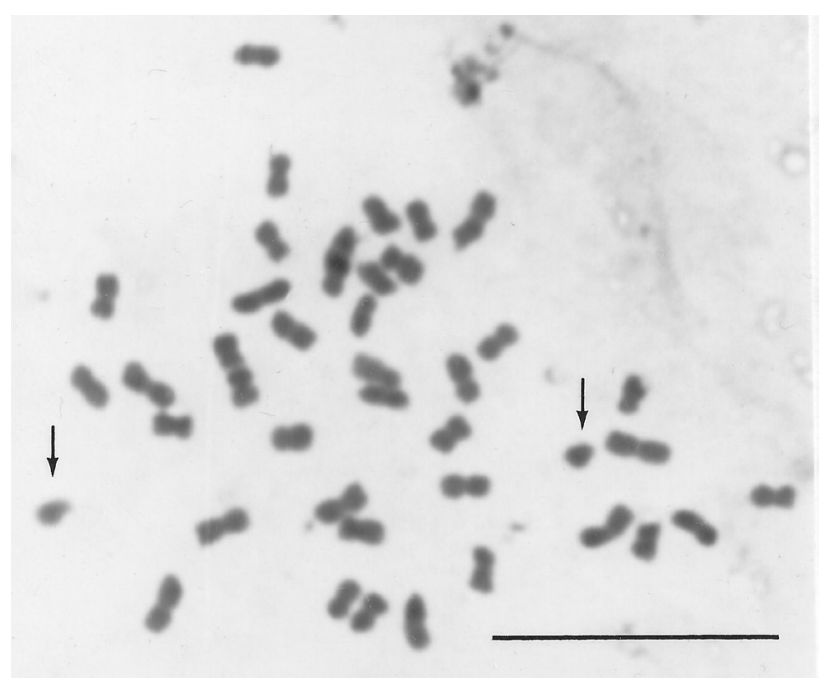

Figura 3. Complemento cromosómico en metafase mitótica de Erythrina americana. Las flechas señalan los cromosomas con satélites. Escala, $10 \mu \mathrm{m}$.

propuesta, pues en los alopoliploides es común el efecto de supresión de la función nucleolar debido a amfiplastía diferencial o dominancia nucleolar (Navashin, 1934; Preuss y Pikaard, 2007). Un mismo origen alopoliploide puede argumentarse en C. grandiflora.

\section{Agradecimientos}

Al Dr. Alfonso Delgado Salinas, la revisión crítica del manuscrito y sus valiosos comentarios, y al Biól. Gabriel Flores Franco, la identificación del ejemplar de Cologania grandiflora.

\section{Literatura citada}

Almada, R. D., J. R. Daviña y J. G. Seijo. 2006. Karyotype analysis and chromosome evolution in southernmost South American species of Crotalaria (Leguminosae). Botanical 
Journal of the Linnean Society 150:329-341.

Atchison, E. 1947. Studies in Leguminosae I: Chromosome numbers in Erythrina L. American Journal of Botany 34:407-414.

Atchison, E. 1950. Studies in the Leguminosae. V. Cytological observations on Crotalaria. Journal of the Elisha Mitchell Scientific Society 66:70-75.

Cotias de Oliveira, A. L. P. y M. L. R. Aguiar-Perecin. 1999. Karyotype evolution in the genus Crotalaria (Leguminosae). Cytologia 64:165-174.

Cruz, N. D., M. R. G. Ungaro y D. M. Medina. 1976. Observaçōes citológicas em três espécies de Erythrina. Bragantha 35:133-139.

Forni-Martins, E. R. y N. D. Cruz. 1996. Recombination indices in species of Erythrina L. (Leguminosae, Papilionoideae). Botanical Journal of the Linnean Society 122:163-170.

Goldblatt, P. 1981. Cytology and the phylogeny of Leguminosae. In Advances in Legume Systematics, R. M. Polhill y P. M. Raven (eds.). Royal Botanical Gardens, Kew. p. 427-463.

Hunziker, J. H., C. C. Xifreda y A. F. Wulff. 1985. Estudios cromosómicos en angiospermas de Sudamérica. Darwiniana 26:7-14.

Jalil, R., M. Pal, G. S. Srivastava y T. N. Khoshoo. 1982. Cytogenetics of Erythrina $\mathrm{x}$ resuparcelli Srivastava. Allertonia 3:19-24.

Kelly, L.M. y A. Delgado Salinas. s/d. Los arboles de la UNAM. Instituto de Biología, UNAM, México, D.F. http://www. arboles.org/arboles_de_la_unam.html; última consulta: 5.VI.2010.

Krukoff, B. A. y R. C. Barneby. 1974. A conspectus of the genus Erythrina. Lloydia 37:332-459.

Lackey, J. A. 1980. Chromosome numbers in the Phaseoleae (Fabaceae: Faboideae) and their relation to taxonomy. American Journal of Botany 67:595-602.

Lee, J. y T. Hymowitz. 2001. A molecular phylogenetic study of the subtribe Glycininae (Leguminosae) derived from the chloroplast DNA rps16 intron sequences. American Journal of Botany 88:2064-2073.

Levan, A., K. Fredga y A. A. Sandberg. 1964. Nomenclature for centromeric position on chromosomes. Hereditas 52:201-219.

Lewis, W. H. 1974. Chromosomes and phylogeny of Erythrina (Fabaceae). Lloydia 37:460-464.

Martínez, M. 1979. Catálogo de nombres vulgares y científicos de plantas mexicanas. Fondo de Cultura Económica, México, D. F. 1247 p.

Musicante, M. L. y L. Galetto. 2008. Biología reproductiva de Cologania broussonetii (Fabaceae, Faboideae). Darwiniana 46:7-16.

Navashin, M. 1934. Chromosomal alterations caused by hybridization and their bearing upon certain general genetic problems. Cytologia 5:169-203.
Neill, D. A. 1988. Experimental studies on species relationships in Erythrina (Leguminosae: Papilionoideae). Annals of the Missouri Botanical Garden 75:886-969.

Palomino, G. y R. Vázquez. 1991. Cytogenetic studies in Mexican populations of species of Crotalaria L. (LeguminosaePapilionoideae). Cytologia 56:343-351.

Poggio, L., S. M. Spert y R. H. Fortunato. 2008. Citogenética evolutiva en leguminosas americanas. Rodriguésia 59:423-433.

Portal de la Reserva Ecológica Pedregal de San Ángel. 2007. http://www.cic-ctic unam.mx:31101/reserva ecológica; última consulta: 5.VI.2010.

Preuss, S. y C. S. Pikaard. 2007. rRNA gene silencing and nucleolar dominance: Insights into a chromosme-scale epigenetic on/off switch. Biochimica et Biophysica Acta 1769:383-392.

Rzedowski, J. 1954. Vegetación del Pedregal de San Ángel (Distrito Federal, México). Anales de la Escuela Nacional de Ciencias Biológicas 8:59-129.

Rzedowski, J. y G. C. de Rzedowski. 1993. Datos sobre la dinámica de la flora fanerogámica del Valle de México, con énfasis en especies nativas raras, en peligro de extinción y aparentemente extintas. Acta Botanica Mexicana 25:81-108.

Sede, S. M., R. H. Fortunato y L. Poggio. 2006. Chromosome evaluation of southern South American species of Camptosema and allied genera (Diocleinae- PhaseoleaePapilionoideae- Leguminosae). Botanical Journal of the Linnean Society 152:235-243.

Seen, H. 1938. Chromosome number relationships in the Leguminosae. Bibliographica Genetica 12:175-336.

Sharma, A. K. 1970. Polyploidy and chromosome size. Chromosomes Today 3:248-252.

Sharma, A. y S. Sen. 2002. Chromosome Botany. Science, Enfield, New Hampshire. 155 p.

Sharma, A. K. y A. Sharma. 1984. Trends in chromosome evolution in the plant kingdom. In Chromosomes in evolution of eukaryotic groups 2, A. K. Sharma (ed.). CRC, New Delhi. p. 227-239.

Sinha, S. S. N. y H. Roy. 1979. Cytological studies in the genus Phaseolus I. Mitotic analysis in fourteen species. Cytologia 44:191-199.

Sousa, M. y A. Delgado. 1998. Leguminosas mexicanas: fitogeografía, endemismo y orígenes. In Diversidad biológica de México. Orígenes y distribución, T. P. Ramamoorthy, R. Bye, A. Lot y J. Fa (comp.). Instituto de Biología, Universidad Nacional Autónoma de México, México, D. F. p. 449-500.

Souza, M. G. C. y A. M. Benko-Iseppon. 2004. Cytogenetics and chromosome banding patterns in Caesalpinioideae and Papilionoideae species of Pará, Amazonas, Brazil. Botanical Journal of the Linnean Society 144:181-191.

Tapia-Pastrana, F y P. Mercado-Ruaro. 2001. A combination of 
the "squash" and "splash" techniques to obtain the karyotype and assess meiotic behavior of Prosopis laevigata L. (Fabaceae: Mimosoideae). Cytologia 66:11-17.
Valiente-Banuet, A. y G. E. Luna. 1990. Una lista florística actualizada para la Reserva del Pedregal de San Ángel, México, D. F. Acta Botanica Mexicana 9:13-30. 